\section{DESEMPENHO E INDICADORES CARDIORRESPIRATÓRIOS EM CRIANÇAS NO TESTE PROGRESSIVO MÁXIMO}

\author{
PERFORMANCEAND CARDIORESPIRATORY INDICATORS IN CHILDREN IN THEMAXIMUM PROGRESSIVE TEST
}

RENDIMIENTO E INDICADORES CARDIORRESPIRATORIOS EN NIÑOS EN PRUEBA INCREMENTAL MÁXIMA

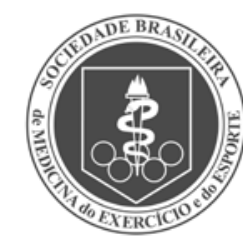

Artigo Original

Original ARTICLE Artículo Original
Rodrigo Vinícius Ferreira ${ }^{1,2}$ (Profissional de Educação Física)

José Carlos Leal1,2

(Profissional de Educação Física)

Marisa Afonso Andrade Brunherotti ${ }^{1}$ (Fisioterapeuta)

1. Universidade de Franca, Franca, São Paulo, Brasil.

2. Centro Universitário de Formiga, Minas Gerais, MG, Brasil.

\section{Correspondência:}

Marisa Afonso Andrade Brunherotti Programa de Pós Graduação Mestrado e Doutorado em Promoção de Saúde da Universidade de Franca. Av. Doutor Armando de Sales Oliveira, 201, Parque Universitário, Franca, SP, Brasil. 14404-600. marisa.brunherotti@unifran.edu.br

\section{RESUMO}

Introdução: As crianças estão sempre envolvidas em atividades físicas e em esportes coletivos. Assim, os estudos que mesclam o desempenho físico e a capacidade cardiorrespiratória são necessários para incentivar e aplicar ações promotoras de saúde. Objetivo: Avaliar a resposta do desempenho físico e dos indicadores cardiorrespiratórios na interrupção espontânea do teste progressivo máximo em crianças. Método: Estudo prospectivo, analítico, observacional e transversal. Participaram 106 crianças com média de idade de 10,2 $\pm 1,1$. O peso e altura foram obtidos para determinar o índice de massa corporal, e a capacidade cardiorrespiratória foi avaliada por meio do teste progressivo máximo de Léger. As variáveis observadas foram: frequência cardíaca, frequência respiratória, pico de fluxo expiratório forçado, volume de oxigênio máximo e força da musculatura abdominal. As crianças foram divididas conforme o desempenho no teste progressivo máximo, grupo de até 280 metros e grupo acima de 280 metros. Resultado: O valor médio do índice de massa corporal foi de 17,8 kg/m² e o volume de oxigênio máximo de $40,5 \mathrm{mlO}_{2} / \mathrm{kg}^{-1}$. $\mathrm{min}^{-1}$. O grupo que atingiu maior distância no teste progressivo máximo obteve menor índice de massa corporal $(p=0,002)$, maior velocidade $(p=0,000)$, maior tempo no esforço físico $(p=0,000)$, maior volume de oxigênio máximo $(p=0,000)$, maior força muscular abdominal $(p=0,007)$. As variáveis frequência cardíaca, respiratória e o peak flow tinham valores esperados para a idade, sem diferença entre os grupos. O grupo que atingiu maior distância teve correlação com a velocidade $(r=0,950, p<0,000)$ e com volume de oxigênio $(r=0,740, p<0,000)$. Já o grupo de menor distância apresentou maior correlação com o índice de massa corporal $(r=-0,475, p=0,000)$ e frequência respiratória $(r=0,585, p<0,674)$. Conclusão: As crianças com menor massa corporal apresentaram melhor desempenho no teste progressivo máximo, e as meninas destacaram-se pelo maior tempo de esforço físico sem aumento do consumo de oxigênio máximo.

Descritores: sistema cardiovascular; sistema respiratório; criança; teste de esforço.

\section{ABSTRACT}

Introduction: Children are always involved in physical activities and team sports. Thus, studies that combine physical performance and cardiorespiratory fitness are necessary to encourage and implement health promotion actions. Objective: To evaluate the response of physical performance and cardiorespiratory indicators in the spontaneous interruption of a maximum progressive test in children. Method: Prospective, analytical, observational and cross-sectional study. One hundred and six children with a mean age of $10.2 \pm 1.1$ years participated in the study. Weight and height were obtained to determine the body mass index and the cardiorespiratory fitness was evaluated by the maximum progressive Léger test. The observed variables were heart rate, respiratory rate, peak forced expiratory flow, maximum oxygen volume, and abdominal muscle strength. The children were divided according to their performance in the maximum progressive test, a group achieving up to 280 meters and a group achieving over 280 meters. Result: The mean value of body mass index was $17.8 \mathrm{~kg} / \mathrm{m}^{2}$ and the maximum oxygen volume was $40.5 \mathrm{~m} / \mathrm{O}_{2} / \mathrm{kg}^{-1}$. $\mathrm{min}^{-1}$. The group that reached the greater distance in the maximum progressive test had a lower body mass index ( $p=0.002)$, a higher speed ( $p=0.000)$, a longer time in the physical effort ( $p=0.000)$, higher maximum oxygen volume $(p=0.000)$, and a greater abdominal muscle strength ( $p=0.007$ ). The variables heart rate, respiratory rate and peak flow had values expected for age with no difference between groups. The group that reached the greater distance had a correlation with speed $(r=0.950, p<0.000)$ and with oxygen volume $(r=0.740, p<0.000)$. In contrast, the shorter distance group had a higher correlation with body mass index $(r=-0.475, p=0.000)$ and respiratory rate $(r=0.585, p<0.674)$. Conclusion: Children with a lower body mass presented better performance in the maximum progressive test, and girls stood out for the greater time of physical effort without increasing the maximum oxygen uptake.

Keywords: cardiovascular system; respiratory system; child; exercise test.

\section{RESUMEN}

Introducción: Los niños siempre están involucrados en actividades físicas y en deportes de equipo. Por lo tanto, se necesitan estudios que combinan el rendimiento físico y la capacidad cardiorrespiratoria para fomentar e implementar acciones que promueven la salud. Objetivo: Evaluar la respuesta del rendimiento físico e indicadores cardiorrespiratorios en la interrupción espontánea del test progresivo máximo en niños. Método: Estudio prospectivo, analítico, observacional y transversal. Participaron 106 niños, con edad promedio de 10,2 $\pm 1,1$. El peso y la altura 
fueron obtenidos para determinar el índice de masa corporal y la capacidad cardiorrespiratoria fue evaluada por la prueba incremental máxima de Léger. Las variables observadas fueron: frecuencia cardiaca, frecuencia respiratoria, pico de flujo espiratorio forzado, volumen máximo de oxígeno y fuerza de los músculos abdominales. Los niños fueron divididos en función del rendimiento en la prueba incremental máxima, un grupo de hasta 280 metros y un grupo de más de 280 metros. Resultados: El índice de masa corporal promedio fue de $17,8 \mathrm{~kg} / \mathrm{m}^{2}$ y el volumen máximo de oxígeno 40,5 $\mathrm{mlO}_{2} / \mathrm{kg}^{-1}$. $\mathrm{min}^{-1}$. El grupo que alcanzó la mayor distancia en la prueba incremental máxima presentó menor índice de masa corporal $(p=0,002)$, mayor velocidad $(p=0,000)$, más tiempo en esfuerzo físico $(p=0,000)$, mayor volumen de oxígeno máximo ( $p=0,000)$, mayor fuerza muscular abdominal ( $p=0,007$. Las variables frecuencia cardiaca, respiratoria y el peak flow tenían los valores esperados para la edad, sin diferencia entre los grupos. El grupo que alcanzó mayor distancia presentó correlación con la velocidad $(r=0,950, p<0,000)$ y con el volumen de oxígeno ( $r=0,740, p<0,000)$. Sin embargo, el grupo de distancia más corta presentó mayor correlación con el índice de masa corporal ( $r=-0,475, p=0,000$ ) y la frecuencia respiratoria ( $r=0,585, p<0,674)$. Conclusión: Los niños con menor masa corporal mostraron mejor rendimiento en la prueba incremental máxima, y las niñas se destacaron con mayor tiempo de esfuerzo físico sin aumento del consumo máximo de oxígeno.

Descriptores: sistema cardiovascular; sistema respiratorio; niño; prueba de esfuerzo.

\section{INTRODUÇÃO}

Experiências motoras vivenciadas pelas crianças e as atividades diárias colaboram para o aprendizado de habilidades motoras de maior complexidade e auxiliam na manutenção do peso corporal' . A redução da prática da atividade física na infância é caracterizada pelo maior envolvimento das crianças em jogos de vídeo game e programas de televisão².

A inatividade coopera para o sedentarismo e para o sobrepeso, da qual maiores demandas do sistema cardiorrespiratório são necessárias para a atividade física. Assim sendo, o consumo de oxigênio é superior e os volumes e capacidades pulmonares ajustam para atender as solicitações metabólicas do organismo ${ }^{3,4}$.

A resposta do desempenho físico pode ser caracterizado através do teste progressivo máximo, que verifica o comportamento de alguns indicadores que relacionam com a capacidade cardiorrespiratória como, o consumo máximo de oxigênio $\left(\mathrm{VO}_{2} \mathrm{máx}\right)^{5}$.

Algumas evidências conceituam que as crianças respondem diferente aos adultos nos indicadores cardiorrespiratório e metabólica durante o teste de esforço progressivo. Portanto, as crianças mostram níveis semelhantes aos adultos de capacidade de esforço físico, mas apresentam maior eficiência metabólica durante o esforço 6 .

Na literatura, grande parte das investigações sobre as respostas cardiorrespiratórias em crianças submetidas ao esforço físico, são voltadas à comparações com indivíduos adultos e algumas diferenças são evidenciadas, já que as fases biológicas são distintas. Desta forma, o objetivo deste estudo foi avaliar a resposta do desempenho físico e dos indicadores cardiorrespiratórios na interrupção espontânea do teste progressivo máximo em crianças.

\section{MÉTODOS}

O estudo foi prospectivo, analítico, observacional e transversal. Parti-

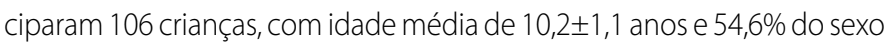
feminino. A seleção da amostra foi não probabilística por conveniência e o estudo obteve a aprovação do Comitê de Ética da Universidade de Franca, protocolo n`35449314.0000.5495. Os pais dos menores assinaram o Termo Consentimento Livre Esclarecido e os participantes da pesquisa assinaram o Termo de Assentimento. Os dados foram obtidos entre os meses de novembro de 2014 e março de 2015, em colégio particular.

Fizeram parte da inclusão, crianças em boas condições de saúde com independência motora, entre oito e 12 anos de idade, do segundo ao sexto ano do ensino fundamental. Para a exclusão foi considerado as crianças que não quiseram participar ou não conseguiram executar todos os testes propostos.

Como parte da avaliação as crianças responderam a ficha de identificação pessoal e o formulário de atividades físicas. Foram coletadas as medidas antropométricas, peso e altura e, para os indicadores cardiorrespiratórios foram obtidos, pico de fluxo expiratório forçado, frequência respiratória, frequência cardíaca, $\mathrm{VO}_{2}$ máx. e o teste de força/resistência da musculatura abdominal.

Para o teste de esforço progressivo máximo, as crianças foram submetidas ao teste de Léger ${ }^{7}$, o qual foi aplicado de forma individual para as crianças, a interrupção do teste ocorreu de modo voluntário. Para o teste de Léger, utilizou-se dois cones com distância de 20 metros e a cada sinal sonoro a criança transpôs pelo menos com um dos pés a linha do cone. O teste constou com 21 estágios, considerando no primeiro estágio uma velocidade de $8,5 \mathrm{~km} / \mathrm{h}$., seguindo o acréscimo de $0,5 \mathrm{~km} / \mathrm{h}$. em cada estágio até a interrupção voluntaria do teste.

Os dados antropométricos foram utilizados para calcular o Índice de Massa Corporal (IMC), sendo classificados através da curva IMC percentil por idade de acordo com a Organização Mundial da Saúde (OMS) ${ }^{8}$. Para a classificação do peso foi considerado: Baixo Peso - IMC percentil entre 3th e 15th; Peso Normal - IMC percentil entre 15th e 85th e Sobrepeso - IMC Percentil maior que 85th.

Para a aferição das frequências cardíacas foi utilizado o monitor cardíaco da marca Polar, modelo FT1. Já a frequência respiratória foi mensurada através da observação da expansão da caixa torácica por um minuto. O pico de fluxo expiratório forçado a partir da capacidade pulmonar total, foi aferido pelo medidor portátil da marca, obtendo o melhor resultado de três medidas, os valores foram ajustados para a idade e altura ${ }^{9}$ e os valores finais foram representados através da porcentagem do previsto considerando para normalidade valor igual ou acima de $80 \%$. Para considerar como resposta de broncoespasmo induzido pelo exercício, o peak flow obteve redução de $20 \%$ a 25\%, diferença entre o valor do pré e pós teste de esforço progressivo máximo ${ }^{10}$.

$\mathrm{O} \mathrm{VO}_{2}$ pico foi obtido com formula indireta ${ }^{7}$, e classificado como, excelente (48,9 ml/Kg.min), bom (47,9 a 38 ml/Kg.min), médio (37,8 a $30,5 \mathrm{ml} / \mathrm{Kg} \cdot \mathrm{min})$, regular (29,9 a 23,1 ml/Kg.min) e fraco (22,9 ml/Kg.min) ${ }^{11}$.

Para a coleta dos dados, as crianças permaneceram na posição ortostática e os dados foram coletados imediatamente após a interrupção voluntaria do teste.

Através da análise da mediana, as crianças foram divididas em dois grupos referente a distância percorrida com o teste de Léger. Um grupo com 
55 crianças que atingiram até 280 metros ( 140 a 280m) e outro grupo com 51 crianças que atingiram acima de 280 metros de distância ( 300 a 880m).

Os dados foram expostos de forma descritiva como média, desvio padrão, valores mínimos e máximos. Foi aplicado o teste de Kolmogorov-Smirnov para avaliar a dispersão ou normalidade de distribuição dos dados. As variáveis foram submetidas ao teste de Mann-Whitneye teste de correlação de Pearson. Foi considerado com diferença estatisticamente significante quando apresentasse $p<0,05$.

\section{RESULTADOS}

As crianças avaliadas apresentaram, idade média de 10,2 $\pm 1,1$ anos e foram classificadas como peso normal e IMC de $17,8 \pm 3 \mathrm{~kg} / \mathrm{m}^{2}$. Entretanto, $12,9 \%$ estavam classificadas como baixo peso e $34,2 \%$ sobrepeso.

As crianças que fizeram parte deste estudo responderam com valores médios esperados para a idade no teste de Léger, na frequência cardíaca e respiratória e no peak flow porcentagem do previsto. Já a força dos músculos abdominais, o valor médio encontrado no grupo ficou abaixo do valor de corte esperado para a idade. A média de $\mathrm{VO}_{2}$ pico nas crianças foi considerado como Bom (Tabela 1).

Entretanto, observou que quando comparou as crianças nas diferentes distâncias percorridas no teste de esforço progressivo máximo, o grupo que atingiu maiores distâncias apresentou valores médios menores no IMC $(p=0,002)$ e na frequência respiratória $(p=0,009)$. Ainda neste grupo, as crianças obtiveram maior força dos músculos abdominais $(p=0,007)$, maior velocidade $(p=0,000)$ e tempo percorrido $(p=0,000)$ no teste de esforço, bem como maior $\mathrm{VO}_{2}$ máx $(p=0,000)$. Já as variáveis, idade, frequência cardíaca e o fluxo expiratório forçado não mostraram diferenças entre os grupos (Tabela 2).

No momento em que as variáveis cardiorrespiratórias foram ajustadas para o sexo, os meninos apresentaram valor médio, menor no tempo do esforço suportado no teste de Léger $(35,4$ segundos, $\mathrm{p}=0,000)$, maior valor no pós teste do peakflow de $10,8 \%(\mathrm{p}=0,044)$, maior valor no pré-teste de $18 \%$ ( $p=0,005$ ) e a força muscular abdominal também foi maior com $3,6$ repetição ( $p=0,020)$, quando comparado com as meninas. As demais variáveis não responderam com diferença estatisticamente significante entre os sexos (Tabela 3).

O grupo que atingiu até $280 \mathrm{mts}$ obteve maior correlação com o IMC com r=-0,475 ( $p=0,000)$ e com a frequência respiratória $r=0,585$ mas sem diferença estatisticamente significante, nas demais variáveis analisadas a correlação foi baixa. No grupo acima de $280 \mathrm{mts}$, a correlação foi alta na velocidade do teste de esforço com $r=0,950(p<0,000)$ e com o VO ${ }_{2}$ máx $(r=0,740, p<0,000)$, as demais variáveis responderam com baixa correlação (Tabela 4).

\section{DISCUSSÃO}

O estudo aos diversos aspectos que permeiam o desempenho físico e a capacidade cardiorrespiratória em crianças, se faz necessário para que ações promotoras de saúde possam ser incentivadas e aplicadas para estimular a atividade física. As crianças desde estudo foram consideradas ativas, além das atividades físicas executadas na escola como atividade curricular, 69,5\% também praticavam atividade física extra escolar orientada e sistematizada como, natação, futebol e dança.
Para avaliar a resposta dos indicadores cardiorrespiratórios das crianças neste estudo utilizou-se o teste de esforço progressivo de Léger? O teste foi selecionado uma vez que se assemelha com a resposta das crianças em atividades físicas ou esportes coletivos praticados na atividade física escolar como: futsal, voleibol, handebol, basquete e futebol. Podendo considerar também modalidades acíclicas, nas quais existem alterações de ritmo, pequenas pausas de recuperação, paradas bruscas e mudanças de direção constantes ${ }^{12,13}$.

Nos indicadores cardiorrespiratórios, verificamos que o $\mathrm{VO}_{2}$ pico nas crianças estavam classificados como "Bom", a resposta pode ter sido alcançada pela rotina semanal de atividade física praticada pelo grupo, além da atividade física na escola no mínimo duas vezes por semana praticavam atividade extra escola. No entanto, o grupo que percorreu distância acima de $280 \mathrm{mts}$ no teste progressivo máximo respondeu com maiores valores do $\mathrm{VO}_{2}$ pico. Este grupo também atingiu, maior velocidade de deslocamento corporal, maior tempo de duração em esforço físico e maior valor de força abdominal. Além disso, estas crianças apresentaram com menor índice de massa corporal.

Tabela 2. Valores médios de idade, IMC e indicadores da capacidade cardiorrespiratória pós-teste das 106 crianças dividas pela distância percorrida.

\begin{tabular}{|c|c|c|c|}
\hline & $\begin{array}{l}\text { Distância percorrida } \\
\text { até } 280 \mathrm{~m} \text { (N 55) }\end{array}$ & $\begin{array}{l}\text { Distância percorrida } \\
\text { acima } 280 \mathrm{~m} \text { (N51) }\end{array}$ & $P$ \\
\hline Idade (anos) & $10,0 \pm 1,2$ & $10,3 \pm 1,1$ & 0,105 \\
\hline IMC $\left(\mathrm{kg} / \mathrm{m}^{2}\right)$ & $18,6 \pm 3,5$ & $16,8 \pm 2,2$ & $0,002^{*}$ \\
\hline $\begin{array}{c}\text { Velocidade TVV } \\
(\mathrm{Km} / \mathrm{h})\end{array}$ & $8,4 \pm 0,12$ & $9,3 \pm 0,57$ & $0,000^{*}$ \\
\hline $\begin{array}{c}\text { Tempo de esforço } \\
\text { (segundos) }\end{array}$ & $104,4 \pm 17,43$ & $189,8 \pm 68,72$ & $0,000^{*}$ \\
\hline $\mathrm{VO}_{2}$ pico $\left(\mathrm{mlO}_{2} / \mathrm{kg} \cdot \mathrm{min}\right)$ & $38,9 \pm 2,5$ & $42,3 \pm 3,0$ & $0,000^{*}$ \\
\hline $\mathrm{FC}(\mathrm{bpm})$ & $189,4 \pm 6,8$ & $189,5 \pm 8,1$ & 0,974 \\
\hline FR (ipm) & $60,4 \pm 2,5$ & $59,0 \pm 2,8$ & $0,009^{*}$ \\
\hline PF \% obtido & $83,7 \pm 25,6$ & $93,5 \pm 28,3$ & 0,067 \\
\hline Força ABD (repetições) & $27,0 \pm 7,3$ & $31,1 \pm 9,1$ & $0,007^{*}$ \\
\hline
\end{tabular}

Teste Mann-Whitney. * - Valores com diferença estatística significativa; N: número de crianças: P: valor de significância; IMC: Indice de Massa Corporal; TVV: Teste de Vai e Vem de 20 metros; ABD: Abdominal; VO2: consumo máximo de oxigênio; FC: frequência cardíaca; PF: peakflow.

Tabela 3. Comparação dos valores médios de idade, IMC e indicadores da capacidade cardiorrespiratória das 106 crianças, conforme o sexo dos participantes do estudo.

\begin{tabular}{c|c|c|c}
\hline & $\begin{array}{c}\text { Sexo feminino } \\
\text { (N57) }\end{array}$ & $\begin{array}{c}\text { Sexo masculino } \\
\text { (N49) }\end{array}$ & $\boldsymbol{P}$ \\
\hline IMC $\left(\mathrm{Kg} / \mathrm{m}^{2}\right)$ & $17,7 \pm 3,0$ & $17,8 \pm 3,1$ & 0,823 \\
\hline Velocidade TWV (Km/h) & $8,8 \pm 0,6$ & $8,9 \pm 0,5$ & 0,208 \\
\hline Tempo de esforço (Segundos) & $189,8 \pm 68,7$ & $154,4 \pm 60,2$ & $0,000^{*}$ \\
\hline VO $_{2}$ pico (mlO $/$ /kg.min) & $40,0 \pm 3,2$ & $41,1 \pm 3,3$ & 0,084 \\
\hline FC pós (bpm) & $189,9 \pm 7,2$ & $189,0 \pm 7,7$ & 0,555 \\
\hline FR pós (ipm) & $59,6 \pm 2,6$ & $59,9 \pm 2,9$ & 0,634 \\
\hline PF \% obtido pós & $100,5 \pm 27,6$ & $118,5 \pm 36,9$ & $0,005^{*}$ \\
\hline PF \% obtido pós & $83,4 \pm 25,1$ & $94,2 \pm 28,7$ & $0,044^{*}$ \\
\hline Força ABD (Repetições) & $27,3 \pm 7,9$ & $30,9 \pm 7,4$ & $0,020^{*}$ \\
\hline
\end{tabular}

Teste Mann-Whitney. * - Valores com diferença estatística significativa; N: número de crianças; P: valor de significância; IMC: Índice de Massa Corporal; TVV: Teste de Vai e Vem de 20 metros; ABD: Abdominal; VO2: consumo máximo de oxigênio; FC: frequência cardíaca; PF: peakflow.

Tabela 1. Valores médios de idade, IMC e indicadores da capacidade cardiorrespiratória dos 106 escolares que participaram do estudo.

\begin{tabular}{|c|c|c|c|c|c|c|c|c|}
\hline & $\begin{array}{l}\text { Idade } \\
\text { (anos) }\end{array}$ & $\begin{array}{c}\text { IMC } \\
\left(\mathrm{Kg} / \mathrm{m}^{2}\right)\end{array}$ & $\begin{array}{c}\text { Velocidade vai } \\
\text { e vem }(\mathrm{Km} / \mathrm{h})\end{array}$ & $\begin{array}{c}\mathrm{VO}_{2} \text { pico } \\
\text { (mlO } / \text { kg.min) }\end{array}$ & $\begin{array}{c}\text { FC } \\
(\mathrm{bpm})\end{array}$ & $\begin{array}{c}\text { FR } \\
\text { (ipm) }\end{array}$ & $\begin{array}{l}\text { PF } \\
(\%)\end{array}$ & $\begin{array}{c}\text { Força ABD } \\
\text { (Rep) }\end{array}$ \\
\hline Média & 10,2 & 17,8 & 8,87 & 40,5 & 189,5 & 59.8 & 88 & 29,1 \\
\hline DP & 1,1 & 3,0 & 0,58 & 3,2 & 7,4 & 2,7 & 27,2 & 7,8 \\
\hline Mínimo & 8 & 13,1 & 8 & 32,6 & 173 & 55 & 36,2 & 7 \\
\hline Máximo & 12 & 26,6 & 11,5 & 51,9 & 210 & 66 & 153,1 & 48 \\
\hline
\end{tabular}


Tabela 4. Correlação dos valores médios de idade, IMC e indicadores da capacidade cardiorrespiratória pós-teste das 106 crianças dividas pela distância percorrida.

\begin{tabular}{|c|c|c|c|c|c|c|c|c|c|}
\hline & & $\begin{array}{l}\text { Idade } \\
\text { (anos) }\end{array}$ & $\begin{array}{c}\text { IMC } \\
\left(\mathrm{kg} / \mathrm{m}^{2}\right)\end{array}$ & $\begin{array}{c}\text { Velocidade } \\
\text { (seg) }\end{array}$ & $\begin{array}{c}\mathrm{VO}_{2} \\
\left(\mathrm{mlO}_{2} / \mathrm{kg} \cdot \min \right)\end{array}$ & $\begin{array}{c}\text { FC } \\
(\mathrm{bpm})\end{array}$ & $\begin{array}{c}\text { FR } \\
\text { (ipm) }\end{array}$ & $\begin{array}{l}\mathrm{PF} \\
\%\end{array}$ & $\begin{array}{c}\text { Força } \\
\text { ABD }\end{array}$ \\
\hline \multirow[t]{2}{*}{$\begin{array}{c}\text { Grupo } \\
(<280 \mathrm{mts})\end{array}$} & r & $-0,071$ & $-0,475$ & 0,261 & 0,120 & 0,073 & 0,585 & 0,039 & $-0,086$ \\
\hline & $p$ & 0,602 & $0,000^{*}$ & 0,051 & 0,375 & 0,597 & 0,674 & 0,775 & 0,526 \\
\hline \multirow[t]{2}{*}{$\begin{array}{c}\text { Grupo } \\
(>280 \mathrm{mts})\end{array}$} & $r$ & 0,169 & 0,142 & 0,950 & 0,740 & 0,077 & $-0,153$ & 0,152 & $-0,154$ \\
\hline & $p$ & 0,230 & 0,314 & $<0,000^{*}$ & $<0,000^{*}$ & 0,590 & 0,281 & 0,286 & 0,273 \\
\hline
\end{tabular}

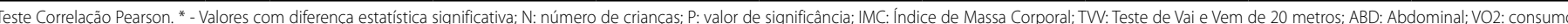
máximo de oxigênio, FC: frequência cardíaca, PF: peakflow.

Estudos científicos apontam que a resposta do $\mathrm{VO}_{2}$ máx.l/min altera com a idade, o incremento ocorre ao longo da infância e, até o inicio da puberdade os meninos apresentam um ligeiro predomínio, mas os valores do $\mathrm{VO}_{2}$ máx são muito semelhantes entre os sexos ${ }^{14}$. Já na puberdade, o valor do $\mathrm{VO}_{2}$ máx para os meninos é, em média, cerca de 25\% maior em comparação com as meninas e, por volta dos 16 anos, a diferença é superior a 50\%. Esta diferença pode ser compreendida devido ao maior desenvolvimento de massa muscular dos meninos, da eficiência cardiorrespiratória, da maturação neural e da maior atividade física voltada aos meninos ${ }^{15,16}$. Neste estudo a média de idade foi de 10 anos e não houve diferença do $\mathrm{VO}_{2}$ pico quando comparado entre os sexos, os meninos responderam somente com $1 \mathrm{mlO}_{2} / \mathrm{Kg}$. mina mais em relação as meninas, porém as meninas atingiram maior tempo de esforço no teste progressivo máximo $\left(35,4\right.$ segundos) com menor $\mathrm{VO}_{2}$ pico.

Em alguns estudos os meninos se destacam com melhor desempenho físico. No grupo de escolares com média de idade de 9,7 anos estudado por Andreasi et al. ${ }^{3}$, os meninos alcançaram melhores resultados e as meninas se destacaram com maior inaptidão física, a qual relacionou com a obesidade e a hiperadiposidade abdominal. Em outro estudo na literatura, o valor médio foi maior nos meninos na distância percorrida $(p<0,05)$ e no pico de consumo de oxigênio máximo $(p<0,05)$, entre crianças de 10 a 12 anos no teste de nove minutos ${ }^{17}$.

Nas crianças avaliadas a frequência cardíaca e respiratória comportaram-se com valores esperados para a idade e os valores foram semelhantes entre as crianças que percorreram diferentes distâncias no teste progressivo máximo, isso mostra que mesmo com menor distância percorrida o sistema cardíaco alcançou o mesmo limiar das crianças que sustentaram o esforço físico por maior tempo.

Crianças e adolescentes apresentam fisiologicamente maiores valores de frequência cardíaca e frequência respiratória basal, assim como no exercício físico progressivo, quando comparado aos valores dos adultos jovens ${ }^{6}$. Essa maior atividade no ritmo cardíaco e respiratório na criança, pode ser explicada morfologicamente, por um mecanismo compensatório que busca amenizar o menor volume do coração e volume sanguíneo, que consequentemente leva a um menor volume de ejeção sistólico, aumentando assim a frequência cardíaca e frequência respiratória ${ }^{18}$.

O pico do fluxo expiratório das crianças mesmo após o esforço físico, alcançou valores esperados para normalidade, demonstrando que as crianças não responderam com reatividade brônquica após o exercício físico. Entretanto, na comparação entre os sexos, os meninos apresentaram no pós exercício maior fluxo aéreo expiratório em relação as meninas. Também a força da musculatura abdominal foi maior nos meninos e para a execução do teste do peakflow é necessário tanto do calibre brônquico quanto da força do grupo muscular abdominal, a qual é envolvida para a execução da expiração forçada.

As nossas crianças responderam com diferença menor de 20\% no fluxo expiratório entre o pré e pós teste, desta forma confirma que o calibre brônquico apresentou na normalidade tanto das meninas quanto dos meninos. Assim a diferença encontrada no fluxo aéreo entre os sexos pode estar mesmo associada à redução da força/resistência abdominal nas meninas e não obstrução brônquica ao fluxo aéreo. Esta diferença da força muscular das meninas pode se dar pela influência das características morfológicas e fisiológicas, próprias do sexo feminino, como: fibras musculares de menor tamanho e quantidade, massa muscular mais baixa e percentual de gordura maior ${ }^{6,19}$.

Um estudo aponta a prevalência de broncoespasmo induzido pelo exercício em crianças obesas, $26 \%$ responderam na avaliação com o peakflow e 23\% deste grupo responderam com a redução do volume expiratório forçado no primeiro segundo, assim verifica a importância da medida do peakflow como parte integrante em programas voltados às crianças ${ }^{20}$. A prevalência da ocorrência de BIE também é observada em crianças com excesso de peso ${ }^{21}$. Foi realizado em um estudo um inquérito transversal em 2941 escolares de 6 a 14 anos e um dos achados foi o BIE em684 crianças (23,3\%), quando comparado as crianças da rede privada e pública, os valores mais elevados de BIE foram os alunos da rede pública de ensino ${ }^{10}$.

Em um estudo foi verificado a relação da força abdominal, da capacidade cardiorrespiratória e o IMC, verificaram que quanto maior o IMC, menor a resistência cardiorrespiratória e menor força abdominal22. Outro estudo encontrou diferença do $\mathrm{VO}_{2}$ máx e da força abdominal entre as crianças com peso normal e sobrepeso. As crianças com sobrepeso apresentaram um percentual de 68\% maior de chance de inaptidão para resistência aeróbica quando comparados com crianças com peso normal, mostrando que as crianças com sobrepeso também tiveram menor desempenho no teste de deslocamento corporal em relação as crianças com peso normal ${ }^{3}$.

As crianças desde estudo que atingiram até 280 metros sinalizou que quanto menor o peso corporal maior distância alcançada. Já o grupo que conseguiu manter o exercício acima de 280 metros estavam com menor índice de massa corpórea e a correlação foi entre a distância percorrida com a velocidade e o $\mathrm{VO}_{2}$ pico atingido pelas crianças, as outras variáveis apresentaram menor correlação. Um estudo realizado avaliou a associação da aptidão cardiorrespiratória e incidência de excesso de peso, o $\mathrm{VO}_{2}$ máx foi maior no sexo masculino ( $p<0,001$ ), e foi também menor nos participantes com sobrepeso e obesidade 4 .

Outra investigação em relação o $\mathrm{VO}_{2}$ máx e a composição corporal, mostrou que $\mathrm{O} \mathrm{VO}_{2}$ máx foi apontado inversamente proporcional à composição corporal ${ }^{12}$, o que corrobora com os achados do presente estudo. Tais dados sugerem que as intervenções visando aumentar a capacidade cardiorrespiratória na primeira infância pode ajudar a inverter as tendências de aumento da obesidade na adolescência e vida adulta.

Em relação aos achados de classificação dos valores médios de IMC percentil por idade, no presente estudo, o grupo foi classificado como peso adequado e envolvidos em atividades físicas na escola e extra escolar, sem predomínio entre os sexos. Alguns autores ${ }^{23,24}$ corroboram 
com estes achados, destacando maior número de crianças classificadas como peso adequado seguido do grupo sobrepeso/obesidade, foi evidenciado que, a prática de atividade física tanto no âmbito escolar quanto fora dele é essencial para a aquisição de hábitos de vida saudáveis, colaborando com a redução do sedentarismo e no combate ao excesso de peso.

No presente estudo pode considerar algumas limitações, as crianças fazem parte de um grupo favorecido socioeconômico e cultural, o qual são incentivados para atividades físicas com acompanhamento especializado na escolar e extra escolar, podendo favorecer a condição física. Além disso, a amostra foi selecionada por conveniência e o resultado retrata um grupo específico.

\section{CONCLUSÃO}

Portanto, com estes resultados podemos concluir que as crianças com maior desempenho físico responderam com maiores valores de consumo máximo de oxigênio e menor peso corporal. As meninas destacaram com maior tempo de esforço, no entanto maiores estudos devem ser dirigidos para determinar as influencias de fatores externos envolvidos neste achado, já que a literatura mostra evidências de melhor resposta dos meninos.

Todos os autores declararam não haver qualquer potencial conflito de interesses referente a este artigo.

CONTRIBUIÇÕES DOS AUTORES: Cada autor contribuiu individual e significativamente para o desenvolvimento do manuscrito. RVS (0000/0002-0852-1973)* contribuiu para a concepção, planejamento, análise, interpretação de dados, elaboração, escrita e aprovação da versão final do manuscrito. JCL (0000-0002-3409-3896)* colaborou na coleta de dados e na aprovação final do manuscrito. MAAB (0000-0002-8058-8523)* contribuiu como orientadora do estudo, planejamento, análise e interpretação dos dados, elaboração do conteúdo e aprovação final do manuscrito. *ORCID (Open Researcher and Contributor ID).

\section{REFERÊNCIAS}

1. Seth A, Sharma R. Childhood obesity. Indian J Pediatr. 2013;80(4):309-17.

2. Fiates GMR, Amboni RDMC, Teixeira E. Comportamento consumidor, hábitos alimentares e consumo de televisão por escolares de Florianópolis. Rev Nutr. 2008;21(1):105-14.

3. Andreasi V, Michelin E, Rinaldi AE, Burini RC. Physical fitness and associations with anthropometric measurements in 7 to 15-year-old school children. J Pediatr (Rio J). 2010;86(6):497-502.

4. Savva SC, Tornaritis MJ, Kolokotroni O, Chadjigeorgiou C, Kourides Y, Karpathios T, et al. High cardiorespiratory fitness is inversely associated with incidence of overweight in adolescence: a longitudinal study. Scand J Med Sci Sports. 2014;24(6):982-9

5. Souza FG, Jaime PJDC, Cunha RM. Teste ergoespirométrico aplicado à prática do exercício físico: um estudo de revisão. Rev Mov. 2013;6(2):481-7.

6. Prado DML, Braga AMF, Rondon MUP, Azevedo LF, Matos LDN, Negrão CE, et al. Comportamento cardiorrespiratório em crianças saudáveis durante o exercício progressivo máximo. Arq Bras Cardiol. 2010;94(4):464-70.

7. Léger LA, Mercier D, Gadoury C. Lambert J. The multistage 20-meter shuttle run test for aerobic fitness. J Sports Sci. 1988;6(2):93-101.

8. Fagundes AA, Barros DC, Duar HA, Sardinha LMV, Pereira MM, Leão MM. Vigilância Alimentar e Nutricional - SISVAN: orientações básicas para a coleta, processamento, análise de dados e informação em serviços de saúde. Brasília, DF: Ministério da Saúde; 2004.

9. Godfrey S, Kamburoff PL, Nairn JR. Spirometry, lung volumes and airway resistance in normal children aged 5 to 18 years. Br J Dis Chest. 1970;64(1):15-24.

10. Teldeschi ALG, Santanna CC, Alres VLT. Prevalência de sintomas respiratórios e condições clínicas associadas à asma em escolares de 6 a 14 anos no Rio de Janeiro. Rev Assoc Med Bras. 2002;48(1):54-9.

11. Matsudo VKR. Testes em ciências do esporte. São Caetano do Sul: Centro de Estudos do Laboratório de Aptidão Física de São Caetano do Sul; 1983.

12. Héroux M, Onywera V,Tremblay MS, Adamo KB, Lopez Taylor J, Jáuregui Ulloa E, et al. The relation between aerobic fitness, muscular fitness, and obesity in children from three countries at different stages of the physical activity transition. ISRN Obes. 2013;2013:134835.
13. Galvani CA, Souza FS, Fonseca FL, Teske M, Sarni RO. Aptidão física aeróbica em crianças e sua relação com obesidade e componentes da síndrome metabólica. Rev Bras Cienc Mov. 2013;21(1):123-31.

14. Silva RJS, Petroski EL. Consumo máximo de oxigênio e estágio de maturação sexual de crianças e adolescentes. Motri. 2008;4(1):13-9.

15. Machado FA, Guglielmo LGA, Denadai BS. Velocidade de corrida associada ao consumo máximo de oxigênio em meninos de 10 a 15 anos. Rev Bras Med Esporte. 2002;8(1):1-6.

16. Armstrong N. Aerobic fitness of children and adolescents. J Pediatr (Rio J). 2006;82(6):406-8.

17. Paludo AC, Batista MB, Faculdade Dom Bosco B, Serassuelo Júnior H, Cyrino ES, Roque ERV. Estimation of cardiorespiratory fitness in adolescents with the 9-minute run/walk test. Rev Bras Cineantropom Desempenho Hum. 2012;14(4):401-8.

18. Vinet A, Nottin S, Lecoq AM, Obert P. Cardiovascular responses to progressive cycle exercise in healthy children and adults. Int J Sports Med. 2002;23(4):242-6.

19. Pollock ML, Gaesser GA, Butcher JD, Després JP, Dishman RK, Franklin BA, et al. The recommended quantity and quality of exercise for developing and maintaining cardiorespiratory and muscular fitness, and flexibility in healthy adults. Med Sci Sports Exerc. 1998;30(6):975-91.

20. Silva LO, Silva PL, Nogueira AMO, Silva MB, Luz GCP, Narciso FV, et al. Avaliação do broncoespasmo induzido pelo exercício avaliado pelo peak flow meter em adolescents obesos. Rev Bras Med Esporte. 2011;17(6):393-6

21. del Río-Navarro B, Cisneros-Rivero M, Berber-Eslava A, Espínola-Reyna G, Sienra-Monge J. Exercise induced bronchospasm in asthmatic and non-asthmatic obese children. Allergol Immunopathol (Madr). 2000;28(1):5-11.

22. Coledam DHC, Batista Junior JP, Glaner MF. Baixa concordância entre os critérios de referência da fitnessgram para adolescentes. Rev Paul Pediatr. 2015;33(2):181-6.

23. Janssen I, Leblanc AG. Systematic review of the health benefits of physical activity and fitness in school-aged children and youth. Int J Behav Nutr Phys Act. 2010;7:40.

24. Jankowski M, Niedzielska A, Brzezinski M, Drabik J. Cardiorespiratory fitness in children: a simple screening test for population studies. Pediatr Cardiol. 2015;36(1):27-32. 